\title{
Tangential Carathéodory-Fejer Interpolation for Stieltjes Functions at Real Points
}

\author{
V. Bolotnikov
}

\begin{abstract}
In this paper we consider a generalization of the classical interpolation problem in a special class of analytic matrix-valued functions. The method used to solve this problem is that of the fundamental matrix inequality, suitably adapted to the present situation.
\end{abstract}

Keywords: Carathéodory-Fejer interpolation, fundamental matrix inequalities

AMS subject classification: Primary 47A57, secondary 30E05

\section{Introduction}

In this paper we consider the tangential Carathéodory-Fejer interpolation problem in the Stieltjes class.

Definition 1.1. A matrix-valued function $w$, holomorphic in the complex plane $\mathbb{C}$ with a cut along the semi-axis $\mathbb{R}_{+}=[0,+\infty)$ is called a Stieltjes function if

$$
\text { 1) } \frac{w(z)-w(z)^{*}}{z-\bar{z}} \geq 0 \quad \text { for } z \neq \bar{z} \quad \text { and } \quad \text { 2) } w(x) \geq 0 \text { for } x<0
$$

The class of all $\boldsymbol{C}^{\boldsymbol{m} \times \boldsymbol{m}}$-valued Stieltjes functions is denoted by $\mathbf{S}_{\boldsymbol{m}}$.

We formulate two theorems which are proved in [18].

Theorem 1.2. The function $w$ belongs to $S_{m}$ if and only if

$$
\frac{w(z)-w(z)^{*}}{z-\bar{z}} \geq 0 \quad \text { and } \quad \frac{z w(z)-\bar{z} w(z)^{*}}{z-\bar{z}} \geq 0 \quad \text { for } z \neq \bar{z} .
$$

Theorem 1.3. The function $w$ belongs to $S_{m}$ if and only if it admits the integral representation

$$
w(z)=A+\int_{0}^{\infty} \frac{d \sigma(t)}{t-z}
$$

where $A \geq 0$ and $d \sigma \geq 0$ is a $a^{m \times m}$-valued measure such that $\int_{0}^{\infty}(1+t)^{-1} d \sigma(t)<\infty$.

In the class $\mathbf{S}_{m}$ we consider the following interpolation problem (IS):

V. Bolotnikov: Ben Gurion Univ. Negev, P.O. Box 653, 84105 Beer Sheva, Israel

ISSN 0232-2064 / \$ 2.50 C Heldermann Verlag Berlin 
(IS) Given a set of matrices $a_{i}, c_{i} \in \mathbb{C}^{s \times m}, \gamma_{i} \in \mathbb{C}^{s \times \Delta}(i=0, \ldots, n)$ and a point $x<0$ find necessary and sufficient conditions which ensure the existence of a function

$$
w(z)=\sum_{k=0}^{\infty}(z-x)^{k} w_{k}
$$

in $\mathbf{S}_{m}$ such that

$$
\sum_{i=0}^{k} a_{k-i} w_{i}=c_{k} \quad \text { and } \quad \sum_{i=0}^{k} \sum_{j=0}^{n} a_{k-i} w_{i+j+1} a_{n-j}^{*}=\gamma_{k} \quad(k=0, \ldots, n)
$$

and describe the set of all functions when these conditions preva il.

Since $w_{k}=w^{(k)}(x) / k !,(1.3)$ are conditions on derivatives of the function $w$ at the point $x$. Note that representation (1.1) implies $w_{k} \geq 0(k=0,1, \ldots)$ because in case of real interpolation points the two-sided interpolation problem coincides with a tangential one. The tangential Carathéodory-Fejer problem can be set in other classes of analytic functions $[3,5-7,10,14]$. In the paper [13] there was considered the non-degenerate case of the non-tangential problem in the Stieltjes class and in [9] its two-sided generalization for simple interpolating points.

Somewhat another interpolation problem in the same class is given in [1]. We also refer to the monographes $[8,11]$ where a number of appraaches to solve some versions of the Nevanlinna-Pick problem have been developed. In the present paper a central role will be played by the method of the fundamental matrix inequality $[12,15-17]$.

The outline of the paper is as follows: .

In Section 2, following ideas of I. Kovalishina and V. Potapov, we establish the system of matrix inequalities for the problem (IS). A necessary condition of the solvability of problem (IS) is the non-negativity of special matrices $K$ and $K_{p}$ defined in (2.12), (2.13). The description of the set of all solutions to the problem (IS) depends on whether $K$ and $K_{p}$ are degenerate or not. In Section 3 we consider the case where $K$ and $K_{p}$ both are strictly positive. The strict positivity of $K$ and $K_{p}$ is a sufficient condition for the problem (IS) to be solvable. The set of all solutions is parametrized by a linear fractional transformation of the form (3.16) with the resolvent matrix $\theta$ of the class W. (see Definition 3.1). Section 4 deals with the degenerate case; we still have a description of all the solutions as a linear fractional transformation which can be obtained by a suitable version of the Schur algorithm.

\section{The fundamental matrix inequalities}

In this section we characterize the solutions to the problem (IS) in terms of a system of matrix inequalities. Descriptions of the set of solutions in terms of the linear fractional transformation will be given in Sections 3 and 4. We begin with a preliminary lemma.

Lemma 2.1. Let the function $w$ be of the class $S_{m}$ and let

$$
w_{k}=\frac{w^{(k)}(x)}{k !} \quad(k=0, \ldots, 2 n+1 ; x<0) .
$$


Then for all $z \in \mathbb{C} \backslash \mathbb{R}_{+}$the inequalities



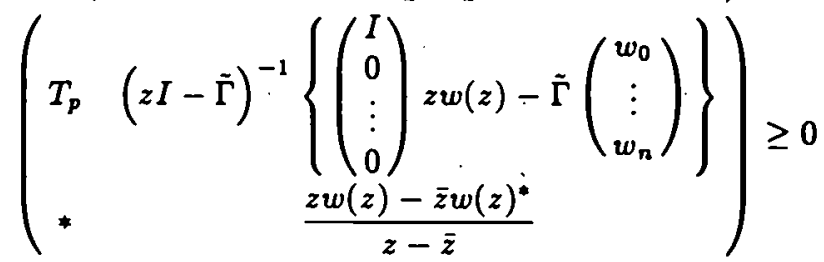

are valid where $T, T_{p} \in \mathbb{C}^{m(n+1) \times m(n+1)}$ are Hankel block matrices defined by

$$
(T)_{i j}=w_{i+j+1} \quad \text { and } .\left(T_{p}\right)_{i j}=x w_{i+j+1}+w_{i+j}
$$

and $\tilde{\Gamma}$ is the $m(n+1) \times m(n+1)$-matrix given by

$$
\tilde{\Gamma}=\left(\begin{array}{cccc}
x I_{m} & & & 0 \\
I_{m} & \ddots & & \\
& \ddots & & \\
0 & & I_{m} & x I_{m}
\end{array}\right) .
$$

Proof: We first establish inequalities (2.2) for the simpliest functions $w \in \mathbf{S}_{m}$ such that a measure $\sigma(t)$ from its integral representation (1.1) has a single point of growth $\mu \geq 0$, i.e. for the function of the form

$$
w(z)=(\mu-z)^{-1} \dot{D}
$$

with some non-negative matrix $D \in \mathbb{C}^{m \times m}$. It follows from (2.1), (2.5) that

$$
w_{k}=(\mu-x)^{-k-1} D \text {. }
$$

Substituting (2.3)-(2.6) into (2.2) we obtain the inequalities

$$
W D W^{*} \geq 0 \quad \text { and } \quad \mu W D W^{*} \geq 0 \text {, }
$$

where

$$
W^{*}=\left((\mu-x)^{-1} I_{m}, \ldots,(\mu-x)^{-n-1} I_{m},(\mu-z)^{-1} I_{m}\right)
$$

which are evidently valid for $\mu \geq 0$ and $D \geq 0$. Hence the inequalities (2.2) hold for the simpliest functions of the class $S_{m}$. After summations and taking a limit we obtain that (2.2) hold for every function of the class $S_{m}$ 
The following theorem characterizes all the solutions to the problem (IS) in terms of a system of matrix inequalities.

Theorem 2.2. Let. $w$ be a $\mathbb{C}^{m \times m}$-valued function analytic in $\mathbb{C} \backslash \mathbb{R}_{+}$. Then $w$ is a solution to the problem (IS) if and only if it satisfies the system of inequalities

$$
\begin{aligned}
\left(\begin{array}{cc}
K & \Gamma(z)(A,-C)\left(\begin{array}{c}
w(z) \\
I_{m}
\end{array}\right) \\
* & \frac{w(z)-w(z)^{*}}{z-\bar{z}}
\end{array}\right) \geq 0 \\
\left(\begin{array}{cc}
K_{p} & \Gamma(z)(A,-\Gamma C)\left(\begin{array}{c}
z w(z) \\
I_{m}
\end{array}\right) \\
* & \frac{z w(z)-\bar{z} w(z)^{*}}{z-\bar{z}}
\end{array}\right) \geq 0
\end{aligned}
$$

for $z \neq \bar{z}$, where

$$
\begin{aligned}
A & =\left(\begin{array}{c}
a_{0} \\
\vdots \\
a_{n}
\end{array}\right), \quad C=\left(\begin{array}{c}
c_{0} \\
\vdots \\
c_{n}
\end{array}\right) \\
\Gamma & =\left(\begin{array}{cccc}
x I_{s} & & 0 \\
I_{s} & \ddots & \\
& \ddots & \\
0 & & I_{s} & x I_{0}
\end{array}\right) \in \mathbb{C}^{s(n+1) \times s(n+1)} \\
\Gamma(z) & =(z I-\Gamma)^{-1}
\end{aligned}
$$

and $K, K_{p}$ are $s(n+1) \times s(n+1)$ matrices defined by

$$
\begin{gathered}
K=\hat{A}\left(\begin{array}{cccc}
c_{1}^{*} & \ldots & c_{n}^{*} & 0 \\
\vdots & & & \\
c_{n}^{*} & & & \vdots \\
0 & \ldots & & 0
\end{array}\right)-\hat{C}\left(\begin{array}{cccc}
a_{1}^{*} & \ldots & a_{n}^{*} & 0 \\
\vdots & & & \\
a_{n}^{*} & & & \vdots \\
0 & \ldots & & 0
\end{array}\right)+\left(\begin{array}{cccc}
0 & \cdots & 0 & \gamma_{0} \\
\vdots & & & \gamma_{1} \\
0 & & & \vdots \\
\gamma_{0} & \gamma_{1} & \cdots & \gamma_{n}
\end{array}\right) \\
K_{p}=\Gamma K+A C^{*}
\end{gathered}
$$

where $\hat{A}$ and $\hat{C}$ are $\mathbb{C}^{o(n+1) \times m(n+1)}$ Toeplitz block matrices defined by

$$
\hat{A}=\left(\begin{array}{cccc}
a_{0} & 0 & \cdots & 0 \\
a_{1} & \ddots & & \vdots \\
\vdots & \ddots & & 0 \\
a_{n} & \cdots & a_{1} & a_{0}
\end{array}\right) \quad \text { and } \quad \hat{C}=\left(\begin{array}{cccc}
c_{0} & 0 & \cdots & 0 \\
c_{1} & \ddots & & \vdots \\
\vdots & \ddots & & 0 \\
c_{n} & \cdots & c_{1} & c_{0}
\end{array}\right)
$$

Proof. Let $w$ be a solution to the problem.(IS). Since $w$ is of the class $\mathbf{S}_{m}$, it satisfies in view of Lemma 2.1 the inequalities (2.2). Multiplying (2.2) by the matrix 
$\left(\begin{array}{cc}\dot{A} & 0 \\ 0 & I_{m}\end{array}\right)$ on the left, by its adjoint on the right and using relations

$$
\hat{A}\left(\begin{array}{c}
I_{m} \\
0 \\
\vdots \\
0
\end{array}\right)=A, \quad \hat{A}\left(\begin{array}{c}
w_{0} \\
w_{1} \\
\vdots \\
w_{n}
\end{array}\right)=C, \quad \hat{A} \tilde{\Gamma}=\Gamma \hat{A}
$$

(which follow immediately from (1.3), (2.4), (2.9), (2.10) and (2.14)) we obtain (2.7), (2.8) with blocks $\hat{A} T \hat{A}^{*}$ and $\hat{A} T_{p} \hat{A}^{*}$ instead of $K$ and $K_{p}$, respectively. But the equality

$$
\hat{A} T \hat{A}^{*}=K
$$

follows from (2.3), (2.12) and interpolation conditions (1.3), and after a multiplication of the evident identity

$$
T_{p}=\tilde{\Gamma} T+\left(\begin{array}{c}
I_{m} \\
0 \\
\vdots \\
0
\end{array}\right)\left(w_{0}^{*}, \ldots, w_{n}^{*}\right)
$$

by $\hat{A}$ on the left and by $\hat{A}^{*}$ on the right we obtain in view of (2.13), (2.15) and (2.16) that $\hat{A} T_{p} \hat{A}^{*}=\Gamma \hat{A} T \hat{A}^{*}+A C^{*}=K_{p}$ which ends the necessary part of the theorem.

Conversely, let assume that a $\mathbb{C}^{m \times m}$-valued function $w$ analytic in $\boldsymbol{C} \backslash \mathbb{R}_{+}$satisfies the system of matrix inequalities (2.7) and (2.8). Then, in particular,

$$
\frac{w(z)-w(z)^{*}}{z-\bar{z}} \geq 0 \quad \text { and } \quad \frac{z w(z)-\bar{z} w(z)^{*}}{z-\bar{z}} \geq 0
$$

for $\dot{z} \neq \bar{z}$. By Theorem 1.2, these inequalities imply that $w$ belongs to $S_{m}$.

The positivity of the matrix-valued function (2.7) implies the boundedness of its non-diagonal block, i.e. of the functions

$$
(z-x)^{-k-1}\left\{\left(\sum_{i=0}^{k} a_{i}(z-x)^{i}\right) w(z)-\sum_{i=0}^{k} c_{i}(z-x)^{i}\right\} \quad(k=0, \ldots, n)
$$

in compact neighbourhoods of the point $x$. Substituting into (2.17) the Taylor expansion (1.2) and setting $z \rightarrow x$ we obtain consequently

$$
\sum_{j=0}^{k} a_{k-j} w_{j}=c_{k} \quad(k=0, \ldots, n) .
$$

Multiplying (2.7), (2.8) by the matrix-valued function $Q(z)=\left(\begin{array}{cc}I_{0(n+1)} & 0 . . \\ -\Gamma(z) & \Gamma(z) A\end{array}\right)$ on the left and by $Q(z)$ on the right we come to inequalities

$$
\left(\begin{array}{cc}
K & W(z) \\
W(z)^{*} & R(z)
\end{array}\right) \geq 0 \text { and } \quad\left(\begin{array}{cc}
K_{p} & W_{p}(z) \\
W_{p}(z)^{*} & R_{p}(z)
\end{array}\right) \geq 0
$$


where

$$
\begin{aligned}
W(z)= & K \Gamma(\bar{z})^{*}+\Gamma(z)\{A w(z)-C\} A^{*} \Gamma(\bar{z})^{*} \\
W_{p}(z)= & -K_{p} \Gamma(\bar{z})^{*}+\Gamma(z)\{z A w(z)-\Gamma C\} A^{*} \Gamma(\bar{z})^{*} \\
R(z)= & \Gamma(\bar{z}) K \Gamma(\bar{z})^{*}-\Gamma(\bar{z}) \Gamma(z)\{A w(z)-C\} A^{*} \Gamma(\bar{z})^{*} \\
& -\Gamma(\bar{z}) A\left\{w(z)^{*} A^{*}-C^{*}\right\} \Gamma(z)^{*} \Gamma(\bar{z})^{*} \\
& +\frac{1}{z-\bar{z}} \Gamma(\bar{z}) A\left\{w(z)-w(z)^{*}\right\} A^{*} \Gamma(\bar{z})^{*} \\
R_{p}(z)= & \Gamma(\bar{z}) K_{p} \Gamma(\bar{z})^{*}-\Gamma(\bar{z}) \Gamma(z)\{z A w(z)-\Gamma C\} A^{*} \Gamma(\bar{z})^{*} \\
& -\Gamma(\bar{z}) A\left\{\bar{z} w(z)^{*} A^{*}-C^{*} \Gamma^{*}\right\} \Gamma(z)^{*} \Gamma(\bar{z})^{*} \\
& +\frac{1}{z-\bar{z}} \Gamma(\bar{z}) A\left\{z w(z)-\bar{z} w(z)^{*}\right\} A^{*} \Gamma(\bar{z})^{*} .
\end{aligned}
$$
Then

Lemma 2.3. Let $W, W_{p}, R, R_{p}$ be matrix-valued functions defined by (2.20)-(2.29).

$$
\begin{aligned}
W_{p}(z) & =z W(z)+K \\
R(z) & =\frac{W(z)-W(z)^{*}}{z-\bar{z}} \\
R_{p}(z) & =\frac{z W(z)-\bar{z} W(z)^{*}}{z-\bar{z}}
\end{aligned}
$$

Proof. Using (2.13), (2.20), (2.21) and the identity

$$
I+\Gamma \Gamma(z)=z \Gamma(z)
$$

we obtain

$$
\begin{aligned}
W_{p}(z) & =-\left(K \Gamma^{*}+C A^{*}\right) \Gamma(\bar{z})^{*}+z \Gamma(z) A w(z) A^{*} \Gamma(\bar{z})^{*}+(I-z \Gamma(z)) C A^{*} \Gamma(\bar{z})^{*} \\
& =K\left(I-z \Gamma(\bar{z})^{*}\right)+z \Gamma(z)(A w(z)-C) A^{*} \Gamma(\bar{z})^{*} \\
& =z W(z)+K
\end{aligned}
$$

To prove (2.25) we use the identity

$$
\Gamma K-K \Gamma^{*}=C A^{*}-A C^{*}
$$

which follows immediately from (2.13). Multiplying (2.28) by $\Gamma(\bar{z})$ on the left, by $\Gamma(\bar{z})^{*}$ on the right and using (2.27) we obtain

$$
(z-\bar{z}) \Gamma(\bar{z}) K \Gamma(\bar{z})^{*}=\Gamma(\bar{z}) K-K \Gamma(\bar{z})^{*}+\Gamma(\bar{z})\left\{A C^{*}-C A^{*}\right\} \Gamma(\bar{z})^{*} .
$$

Substituting (2.29) into relation (2.23) and taking into account the resolvent identity 


$$
\begin{aligned}
\Gamma(z)-\Gamma(\bar{z})= & (\bar{z}-z) \Gamma(\bar{z}) \Gamma(z) \text { we obtain } \\
R(z)= & (z-\bar{z})^{-1}\left\{\Gamma(\bar{z}) K-K \Gamma(\bar{z})^{*}+(\Gamma(z)-\Gamma(\bar{z}))(A w(z)-C) A^{*} \Gamma\left(\bar{z}^{*}\right)\right. \\
& +\Gamma(\bar{z}) A\left(w(z)^{*} A^{*}-C^{*}\right)\left(\Gamma(\bar{z})^{*}-\Gamma(z)^{*}\right) \\
& \left.+\Gamma(\bar{z}) A\left(w(z)-w(z)^{*}\right) A^{*} \Gamma(\bar{z})^{*}\right\} \\
= & (z-\bar{z})^{-1}\left\{\Gamma(\bar{z}) K-K \Gamma(\bar{z})^{*}+\Gamma(z)(A w(z)-C) A^{*} \Gamma(\bar{z})^{*}\right. \\
& \left.-\Gamma(\bar{z}) A\left(w(z)^{*} A^{*}-C^{*}\right) \Gamma(z)^{*}\right\} \\
& \frac{W(z)-W(z)^{*}}{z-\bar{z}}
\end{aligned}
$$

By the same way, with help of the identity $\Gamma K_{p}-K_{p} \Gamma^{*}=\Gamma C A^{*}-A C^{*} \Gamma^{*}$ which follows from (2.16), we obtain

$$
R_{p}(z)=\frac{W_{p}(z)-W_{p}(z)^{*}}{z-\bar{z}}=\frac{z W(z)-\bar{z} W(z)^{*}}{z-\bar{z}},
$$

which ends the proof of the lemma

In view of (2.24)-(2.26) the inequalities (2.19) can be rewritten as

$$
\left(\begin{array}{cc}
K & W(z) \\
W(z)^{*} & \frac{W(z)-W(z)^{*}}{z-\bar{z}}
\end{array}\right) \geq 0 \text { and }\left(\begin{array}{cc}
K_{p} & z W(z)+K \\
\bar{z} W(z)^{*}+K & \frac{z W(z)-\bar{z} W(z)}{z-\bar{z}}
\end{array}\right) \geq 0
$$

and imply

$$
\frac{W(z)-W(z)^{*}}{z-\bar{z}} \geq 0 \quad \text { and } \quad \frac{z W(z)-\bar{z} W(z)^{*}}{z-\bar{z}} \geq 0
$$

for $z \neq \bar{z}$. By Theorem 1.2,W is of the class $\mathbf{S}_{(n+1)}$, and therefore is analytic in some neighborhood of $x$. Substituting into (2.20) the Taylor expansion (1.2) of $w$ and using (2.15) and (2.18) we obtain

$$
\begin{aligned}
& W(z)=K \Gamma(\bar{z})^{*}+\hat{A} \Gamma(z)\left(\begin{array}{c}
w(z)-w_{0} \\
-w_{1} \\
\vdots \\
-w_{n}
\end{array}\right) A^{*} \Gamma(\bar{z})^{*} \\
& =-K \Gamma(\bar{z})^{*}+\hat{A}\left(\begin{array}{c}
w_{1}+w_{2}(z-x)+\ldots \\
w_{2}+w_{3}(z-x)+\ldots \\
\vdots \\
w_{n+1}+w_{n+2}(z-x)+\ldots
\end{array}\right) A^{*} \Gamma(\bar{z})^{*} \\
& =-K \Gamma(\bar{z})^{*}+\hat{A}\left(\begin{array}{c}
w_{1}+w_{2}(z-x)+\ldots \\
\vdots \\
w_{n+1}+w_{n+2}(z-x)+\ldots
\end{array}\right)\left(\frac{I_{m}}{(z-x)}, \ldots, \frac{I_{m}}{(z-x)^{n+1}}\right) \hat{A}^{*} \\
& =-K \Gamma(\bar{z})^{*}+\hat{A}\left(\begin{array}{ccc}
w_{1} & \ldots & w_{n+1} \\
\vdots & & \vdots \\
w_{n+1} & \cdots & w_{2 n+1}
\end{array}\right) \hat{A}^{*} \Gamma(\bar{z})^{*}+O(|z-x|) \text {. }
\end{aligned}
$$


Setting $z \rightarrow x$ in (2.30) we obtain in view of analyticity of $W$

$$
K=\hat{A}\left(\begin{array}{ccc}
w_{1} & \ldots & w_{n+1} \\
\vdots & & \vdots \\
w_{n+1} & \cdots & w_{2 n+1}
\end{array}\right) \hat{A}^{*}
$$

Substituting (2.12) and (2.14) into (2.31) and comparing there $s$ right rows we obtain the equality

$$
\left(\begin{array}{c}
\gamma_{0} \\
\vdots \\
\gamma_{n}
\end{array}\right)=\hat{A}\left(\begin{array}{ccc}
w_{1} & \ldots & w_{n+1} \\
\vdots & & \vdots \\
w_{n+1} & \ldots & w_{2 n+1}
\end{array}\right) \cdot\left(\begin{array}{c}
a_{n}^{*} \\
\vdots \\
a_{0}^{*}
\end{array}\right)
$$

which implies

$$
\gamma_{k}=\sum_{i=0}^{k} \sum_{j=0}^{n} a_{k-i} w_{i+j+1} a_{n-j}^{*} \quad(k=0, \ldots, n) .
$$

Equalities (2.19) and (2.33) mean that $w$ is a solution to the problem (IS), which ends the proof of theorem

Setting in Theorem $2.2 s=m, a_{0} \doteq I_{m}, a_{i}=0$ for $i>0$ and $c_{i}=w_{i}(i=$ $0, \ldots, 2 n+1$ ) we obtain the following inversion of Lemma 2.1.

Corollary 2.4. Let the $\mathbb{C}^{m \times m}$-valued function $w$ analytic in $\boldsymbol{C} \backslash \mathbb{R}$ satisfy the inequalities (2.2). Then $w$ belongs to $S_{m}$ and $w^{(k)}(x) / k !=w_{k}(k=0, \ldots, 2 n+1)$.

Remark 2.5. It follows from (2.7), (2.8) that the non-negativity of matrices $K$ and $K_{p}$ is a necessary condition to ensure that the problem (IS) has a solution.

-As will be proved in Section 3 , the condition $\left(\begin{array}{cc}K & 0 \\ 0 & K_{p}\end{array}\right)>0$ is a sufficient one. The matrices $K$ and $K_{p}$ will be called the information matrices of the problem (IS).

\section{Solution to the problem (IS) : the non-degenerate case}

In this section we suppose that the information matrices $K$ and $K_{p}$ are strictly positive and describe the set of all solutions to the problem (IS) under this hypothesis To begin with we recall the necessary definitions .

Definition 3.1. A $d^{2 m \times 2 m}$-valued meromorphic function $\theta$ is of the class $W_{*}$ if

$$
\theta(z) J \Theta(z)^{*}=J \quad(z \in \mathbb{R}) \quad \text { and } \quad \Theta(z) J \Theta(z)^{*} \geq J \quad\left(z \in \mathbb{C}_{+}\right)
$$

and

$$
\Theta(x) J_{\pi} \Theta(x)^{*} \geq J_{\pi} \quad(x<0)
$$

where

$$
J=\left(\begin{array}{cc}
0 & i I_{m} \\
-i I_{m} & 0
\end{array}\right) \quad \text { and } \quad J_{\pi}=\left(\begin{array}{cc}
0 & I_{m} \\
I_{m} & 0
\end{array}\right)
$$

and is of the class $W$ if it satisfies only conditions (3.1). 
Lemma 3.2 (see [12]). The classes $W$ and $W$, are closed under multiplication:

(i) Let $\theta_{1}, \theta_{2} \in W$, and $\theta=\theta_{1} \theta_{2}$. Then, $\theta \in W$, and, for $i=1,2$,

$$
\begin{array}{ll}
\theta(z) J \theta(z)^{*} \geq \theta_{i}(z) J \theta_{i}(z)^{*} & \left(z \in C_{+}\right) \\
\theta(x) J_{\pi} \theta(x) \geq \theta_{i}(x) J \theta_{i}(x)^{*} & (x<0) .
\end{array}
$$

(ii) If $\theta_{1}, \theta_{2} \in \mathbf{W}$, then $\theta=\theta_{1} \theta_{2} \in \mathbf{W}$ and the first inequalities of (9.9) hold.

The following theorem establishes the connection between classes $\mathbf{W}$ and $\mathbf{W}$.. if

Theorem 3.3 (see [13]). The $d^{2 m \times 2 m}$-valued function $\theta$ belongs to $W$, if and only

$$
\theta \in W \quad \text { and } \quad \theta_{p}(z)=P(z) \Theta(z) P(z)^{-1} \in W
$$

where

$$
P(z)=\left(\begin{array}{cc}
z I_{m} & 0 \\
0 & I_{m}
\end{array}\right)
$$

The following two lemmas which in fact are contained in [12] describe a number of functions of the classes $\mathbf{W}$ and $\mathbf{W}$.

Lemma 3.4. Let $H$ be a strictly positive $m \times m$ matrix which is a solution of the Lyapunov equation $\Gamma H-H \Gamma^{*}=-i G J G^{*}$, where $J$ is a matrix defined in (9.2), $G \in \mathbb{C}^{r \times m}$ and $\Gamma \in \mathbb{C}^{m \times m}$. Then the $\mathbb{C}^{2 m \times 2 m}$-valued function $\hat{\theta}(z)=I+i G^{*}(z I-$ $\left.\Gamma^{*}\right)^{-1} H^{-1} G J$ is of the class $\mathbf{W}$ and

$$
\hat{\theta}(z) J \hat{\Theta}(w)^{*}-J=i(\bar{w}-z) G^{*}\left(z I-\Gamma^{*}\right)^{-1} H^{-1}(\bar{w} I-\Gamma)^{-1} G .
$$

Lemma 3.5. Let $H_{1}, H_{2}$ be strictly positive $m \times m$ matrices such that

$$
H_{2}-\Gamma H_{1}^{*}=G_{2} G_{1}^{*}
$$

for some matrices $G_{1}, G_{2} \in \mathbb{C}^{r \times m}$ and $\Gamma \in \mathbb{C}^{m \times m}$. Then the $\mathbb{C}^{2 m \times 2 m}$-valued function

$$
\Theta(z)=I+i\left(\begin{array}{cc}
G_{1}^{*} & G_{1}^{*} \Gamma^{*} \\
G_{2}^{*} & z G_{2}^{*}
\end{array}\right)\left(\begin{array}{cc}
\Gamma(\bar{z})^{*} H_{1}^{-1} G_{1} & 0 \\
0 & \Gamma(\bar{z})^{*} H_{2}^{-1} G_{2}
\end{array}\right) J
$$

(where $\Gamma(z)$ is defined by (2.11)) is. of the class W, and

$$
\begin{gathered}
\Theta(z) J \Theta(w)^{*}-J=i(\bar{w}-z)\left(\begin{array}{c}
G_{1}^{*} \\
G_{2}^{*}
\end{array}\right) \Gamma(\bar{z})^{*} H_{1}^{-1} \Gamma(\bar{w})\left(\dot{G}_{1}, G_{2}\right) \\
\Theta_{p}(z) J \Theta_{p}(w)^{*}-J=i(\bar{w}-z)\left(\begin{array}{c}
G_{1}^{*} \Gamma^{*} \\
G_{2}^{*}
\end{array}\right) \Gamma(\bar{z})^{\bullet} H_{2}^{-1} \Gamma(\bar{w})\left(\Gamma G_{1}, G_{2}\right)
\end{gathered}
$$

Note that under assumption (3.6) the function $\theta$ admits the following representation which can be checked by a direct computation:

$$
\theta(z)=\left\{I+i\left(\begin{array}{c}
G_{1}^{*} \\
G_{2}^{*}
\end{array}\right) \Gamma(\bar{z})^{*} H_{1}^{-1}\left(G_{1}, G_{2}\right) J\right\}\left(\begin{array}{cc}
I & 0 \\
G_{2}^{*} H_{2}^{-1} G_{2} & I
\end{array}\right)
$$


where the first. factor is the function of the class $W$ and the second one is a $J$-unitary matrix. In view of (2.16) matrices

$$
H_{1}=K, \quad H_{2}=K_{p}, \quad G_{1}=C, \quad G_{2}=A
$$

satisfy the conditions of Lemma 3.5 and hence, the $a^{2 m \times 2 m}$-valued function

$$
\Theta(z)=I+i\left(\begin{array}{cc}
C^{*} & 0 \\
0 \& A^{*} & \cdots
\end{array}\right)\left(\begin{array}{cc}
\Gamma(\bar{z})^{*} & \Gamma^{*} \Gamma(\bar{z})^{*} \\
\Gamma(\bar{z})^{*} & z \Gamma(\bar{z})^{*}
\end{array}\right)\left(\begin{array}{cc}
K^{-1} & 0 \\
0 & K_{p}^{-1}
\end{array}\right)\left(\begin{array}{cc}
C & 0 \\
0 & A
\end{array}\right) J
$$

is of the class $W$, and

$$
\begin{gathered}
\Theta(z) J \Theta(w)^{*}-J=i(\bar{w}-z)\left(\begin{array}{c}
C^{*} \\
A^{*}
\end{array}\right) \Gamma(\bar{z})^{*} K^{-1} \Gamma(\bar{w})(C, A) \\
\Theta_{p}(z) J \Theta_{p}(w)^{*}-J=i(\bar{w}-z)\left(\begin{array}{c}
C^{*} \Gamma^{*} \\
A^{*}
\end{array}\right) \Gamma(\bar{z})^{*} K_{p}^{-1} \Gamma(\bar{w})(\Gamma C, A) .
\end{gathered}
$$

In view of (3.7) $\Theta$ admits a representation

$$
\Theta(z)=\hat{\Theta}(z)\left(\begin{array}{ccc}
I & \cdots & 0 \\
A^{*} K_{p}^{-1} & A & I
\end{array}\right)
$$

where the function

$$
\hat{\Theta}(z)=I+i\left(\begin{array}{c}
C^{*} \\
A^{*}
\end{array}\right) \Gamma(\bar{z})^{*} K_{i}^{-1}(C, A) J .
$$

belongs, in view of (2.28) and Lemma 3.4, to the class W. Since $\theta$ and $\theta_{p}$ are both $J$-unitary on the real axis, the symmetry relations

$$
\Theta^{-1}(z)=J \Theta(\bar{z})^{*} J \quad \text { and } \quad \Theta_{p}^{-1}(z)=J \Theta_{p}(\bar{z})^{*} J
$$

hold and together with (3.9) imply

$$
\begin{aligned}
J-\Theta(z)^{-*} J \Theta^{-1}(z) & =J\left(J-\Theta(\bar{z}) J \Theta(\bar{z})^{*}\right) J \\
& =i(\bar{z}-z)\left(\begin{array}{c}
A^{*} \\
-C^{*}
\end{array}\right) \Gamma(z)^{*} K^{-1} \Gamma(z)(A,-C) \\
J-\Theta_{p}(z)^{-*} J \Theta_{p}^{-1}(z) & =i(\bar{z}-z)\left(\begin{array}{c}
A^{*} \\
-C^{*} \Gamma^{*}
\end{array}\right) \Gamma(z)^{*} K_{p}^{-1} \Gamma(z)(A,-\Gamma C) .
\end{aligned}
$$

Since $K, K_{p}>0$, inequalities (2.7), (2.8) are equivalent to the following ones:

$$
\begin{gathered}
\left(w(z)^{*}, I\right)\left\{\frac{J}{i(\bar{z}-z)}-\left(\begin{array}{c}
A^{*} \\
-C^{*}
\end{array}\right) \Gamma(z)^{*} K^{-1} \Gamma(z)(A,-C)\right\}\left(\begin{array}{c}
w(z) \\
I
\end{array}\right) \geq 0 \\
\left(\bar{z} w(z)^{*}, I\right)\left\{\frac{J}{i(\bar{z}-\bar{z})}-\left(\begin{array}{c}
A^{*} \\
-C^{*} \Gamma^{*}
\end{array}\right) \Gamma(z)^{*} K_{p}^{-1} \Gamma(z)(A,-\Gamma C)\right\}\left(\begin{array}{c}
z w(z) \\
I
\end{array}\right) \geq 0
\end{gathered}
$$


which in view of (3.11), (3.12) can be rewritten as

$$
\begin{gathered}
\left(w(z)^{*}, I\right) \frac{\Theta(z)^{-*} J \theta^{-1}(z)}{i(\bar{z}-z)}\left(\begin{array}{c}
w(z) \\
I
\end{array}\right) \geq 0 \\
\left(\bar{z} w(z)^{*}, I\right) \frac{\Theta_{p}(z)^{-*} J \Theta_{p}^{-1}(z)}{i(\bar{z}-z)}\left(\begin{array}{c}
z w(z) \\
I
\end{array}\right) \geq 0 .
\end{gathered}
$$

Using (3.4) and (3.5) one can express the last inequality in the form

$$
\left(w(z)^{*}, I\right) \frac{\Theta(z)^{-*} P(z)^{*} J P(z) \theta^{-1}(z)}{i(\bar{z}-z)}\left(\begin{array}{c}
w(z) \\
I
\end{array}\right) \geq 0 .
$$

The description of all solutions of the system of inequalities (3.13),(3.14) for the non-tangential problem (IS) was obtained in [12]. A generalization of this result to the two-sided problem with simple points of interpolation is given in [9].

The presence of multiple points has no influence on the character of the description, and the same arguments lead to a similary description for the present problem, that's why the proof of Theorem 3.7 will be omitted. To formulate this theorem we need some definitions.

Definition 3.6. Let $\{p, q\}$ be a pair of $\mathbb{C}^{m \times m}$-valued functions meromorphic in $\boldsymbol{C} \backslash \mathbb{R}_{+}$.

(i) $\{p, q\}$ is called a Stieltjes pair if

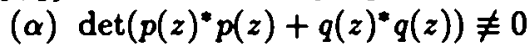

(B) $\left(q(z)^{*} p(z)-p(z)^{*} q(z)\right) /(z-\bar{z}) \geq 0$ for $\operatorname{Im} z \neq 0$

(y) $\left(z q(z)^{*} p(z)-\bar{z} p(z)^{*} q(z)\right) /(z-\bar{z}) \geq 0$ for $\operatorname{Im} z \neq 0$.

(ii) $\{p, q\}$ is said to be equivalent to the pair $\left\{p_{1}, q_{1}\right\}$ if there exists a $a^{m \times m}$-valued function $\Omega(\operatorname{det} \Omega(z) \not \equiv 0)$ meromorphic in $C \backslash \mathbb{R}_{+}$such that $p_{1}=p \Omega$ and $q_{1}=q \Omega$.

The set of all Stieltjes pairs will be denoted by $\overline{\mathbf{S}}_{m}$.

Using the matrix $A$ defined in (2.9) we introduce the following subset of $\overline{\mathbf{S}}_{m}$ :

$$
\overline{\mathbf{S}}_{m}^{0}=\left\{\{p, q\} \in \overline{\mathbf{S}}_{m}: \operatorname{det}\left(p(z)^{*} A^{*} A p(z)+q(z)^{*} q(z)\right) \not \neq 0\right\}
$$

Theorem 3.7. Under the hypothesis $K, K_{p}>0$, let $\theta$ be the function defined by (S.10) and let $\Theta=\left(\theta_{i j}\right)$ be the block decomposition of $\Theta$ into four $\boldsymbol{C}^{m \times m}$-valued functions. Then the linear fractional transformation

$$
w(z)=\left(\theta_{11}(z) p(z)+\theta_{12}(z) q(z)\right)\left(\theta_{21}(z) p(z)+\theta_{22}(z) q(z)\right)^{-1}
$$

gives a parametrization of all solutions to the problem (IS) (or, equivalently, of the system (9.19), (9.14)) when the parameter $\{p, q\}$ varies in $\overline{\mathbf{S}}_{m}^{0}$.

More precisely: $\overline{\mathbf{S}}_{\mathbf{m}}^{\mathbf{0}}$.

(i) Every solution $w$ to the problem (IS) is of the form (9.16) for some pair $\{p, q\} \in$ 
(ii) For every pair $\{p, q\} \in \overline{\mathbf{S}}_{m}^{0}$ the transformation (\$.16) is well defined (the relation $\operatorname{det}\left(\theta_{21}(z) p(z)+\theta_{22}(z) q(z)\right) \not \equiv 0$ is true) and the ${C^{m \times m}}^{m \text { valued function } w}$ defined by (9.16) is a solution to the problem (IS).

(iii) Pairs $\{p, q\},\left\{p_{1}, q_{1}\right\} \in \overline{\mathbf{S}}_{m}^{0}$ are equivalent if and only if they lead under the transformation (\$.16) to the same $w$.

Corollary 3.8. Since the class $\overline{\mathbf{S}}_{m}^{0}$ defined in (9.15) is non-empty, the conditions $K, K_{p}>0$ are sufficient to ensure the problem (IS) to be solvable.

Definition 3.9. The matrix of the linear fractional transformation describing all the solutions of the interpolation problem is called the resolvent matrix of this problem.

To conclude this section we note that the problem (IS) can be set in the class $\bar{S}_{m}$ of Stieltjes pairs ; we consider the following problem (ISP):

(ISP) Given a set of matrices $a_{i}, c_{i} \in \mathbb{C}^{* \times m}, \gamma_{i} \in \mathbb{C}^{\bullet \times \bullet}(i=0, \ldots, n)$ and a point $x<0$, describe all Stieltjes pairs $\{p, q\} \in \overline{\mathbf{S}}_{m}$ with Taylor expansions

$$
p(z)=\sum_{k=0}^{\infty}(z-x)^{k} p_{k} \quad \text { and } \quad q(z)=\sum_{k=0}^{\infty}(z-x)^{k} q_{k}
$$

such that

$$
\sum_{i=0}^{k} a_{k-i} p_{i}=\sum_{i=0}^{k} c_{k-i} q_{i}
$$

and

$$
\sum_{i=0}^{k} \sum_{j=0}^{n}\left(a_{k-i} p_{i+j+1}-c_{k-i} q_{i+j+1}\right) g_{n-j}^{*}=\gamma_{k} \quad(k=0, \ldots, n)
$$

where $\left(g_{0}, \ldots, g_{n}\right) \in \mathbb{C}^{\circ \times m(n+1)}$ is the $u$ nique solution of the system

$$
\sum_{i=0}^{k} p_{i} g_{k-i}^{*}=c_{k}^{*}, \quad \sum_{i=0}^{k} q_{i} g_{k-i}^{*}=a_{k}^{*} . \quad(k=0, \ldots, n) .
$$

Similary interpolation problem on the set of Nevanlinna pairs was considered in $[1,10]$ and in much more general classes in [3]). The following theorem shows that problem (ISP) is also equivalent to a system of matrix inequalities.

Theorem 3.10. A pair $\{p, q\}$ of $\mathbb{C}^{m \times m}$-valued functions meromorphic in $\mathbb{C} \backslash \mathbb{R _ { + }}$ is a solution to the problem (ISP) if and only if it satisfies the inequalities

$$
\left(\begin{array}{cc}
K & \Gamma(z)(A,-C)\left(\begin{array}{l}
p(z) \\
q(z)
\end{array}\right) \\
* & \frac{q(z)^{*} p(z)-p(z)^{*} q(z)}{z-\bar{z}}
\end{array}\right) \geq 0, \quad\left(\begin{array}{cc}
K_{p} & \Gamma(z)(A,-\Gamma C)\left(\begin{array}{l}
p(z) \\
q(z)
\end{array}\right) \\
* & \frac{z q(z)^{*} p(z)-\bar{z} p(z) q(z)}{z-\bar{z}}
\end{array}\right) \geq 0
$$


for $z \neq \bar{z}$, where the matrices $K, K_{p}, A, C, \Gamma$ are as in (2.9), (2.10), (2.12) and (2.19).

A representation of inequalities (3.17) as

$$
\begin{gathered}
\left(p(z)^{*}, q(z)^{*}\right) \frac{\Theta(z)^{-*} J \theta^{-1}(z)}{i(z-\bar{z})}\left(\begin{array}{l}
p(z) \\
q(z)
\end{array}\right) \geq 0 \\
\left(p(z)^{*}, q(z)^{*}\right) \frac{\Theta(z)^{-*} P(z)^{*} J P(z) \Theta^{-1}(z)}{i(z-\bar{z})}\left(\begin{array}{l}
p(z) \\
q(z)
\end{array}\right) \geq 0
\end{gathered}
$$

(analogue of (3.13), (3.14)) with $P, \Theta$ defined in (3.5), (3.8) leads to the following "projectiven analogue of Theorem 3.7 .

Theorem 3.11. Under the hypothesis $K, K_{p}>0$ the following statements are true: formation

(i) All the solutions $\{p, q\}$ of the system (9.17) are parametrized by the linear trans-

$$
\left(\begin{array}{l}
p(z) \\
q(z)
\end{array}\right)=\theta(z)\left(\begin{array}{l}
\tilde{p}(z) \\
\tilde{q}(z)
\end{array}\right)
$$

with the resolvent matrix $\theta \in \mathrm{W}$, defined by (9.10) and parameters $\{\tilde{p}, \tilde{q}\}$ varying in $\overline{\mathbf{S}}_{m}$.

(ii) Pairs $\{p, q\}$ and $\{u, v\}$ are equivalent if and only if the corresponding parameters $\{\tilde{p}, \tilde{q}\}$ and $\{\tilde{u}, \tilde{v}\}$ of the linear transformation (9.18) are equivalent.

Note that the subclass $\overline{\mathbf{S}}_{m}^{0}$ does not appear in Theorem 3.11, since the non-singularity of $q$ in "projective" case have not to be insured.

\section{The Schur algorithm to the degenerate case}

The problem (IS) as a number of classical interpolation problems can be solved by a recursive algorithm. This originates with the work of Schur [19]. We also refer to papers $[4,16]$ where some generalizations of this recursion were considered.

In this section we construct a suitable version of the Schur algorithm to the degenerate case of the problem (IS) where information blocks $K, K_{p}$ being possibly singular: $K, K_{p} \geq 0$. As usual, the Schur algorithm will be concluded in a gradual (step by step) decrease of a number of interpolation conditions both with a simaltaneous recounting of the interpolation data.

Let a pair $\left\{p_{l}, q_{l}\right\} \in \overline{\mathbf{S}}_{m}$ satisfy the following system of matrix inequalities:

$$
\begin{aligned}
& R^{(l)}=\left(\begin{array}{cc}
K^{(l)} & \Gamma_{l}(z)\left(A^{(l)},-C^{(l)}\right)\left(\begin{array}{c}
p_{l}(z) \\
q_{l}(z)
\end{array}\right) \\
* & \frac{q_{l}(z)^{*} p_{l}(z)-p_{l}(z)^{*} q_{l}(z)}{z-\bar{z}}
\end{array}\right) \geq 0 \\
& R_{\mathrm{p}}^{(l)}=\left(\begin{array}{cc}
K_{p}^{(l)} & \Gamma_{l}(z)\left(A^{(l)},-\Gamma_{l} C^{(l)}\right)\left(\begin{array}{l}
p_{l}(z) \\
q_{l}(z)
\end{array}\right) \\
* & \frac{z q_{l}(z)^{*} p_{l}(z)-\bar{z} p_{l}(z) q_{l}(z)}{z-\bar{z}}
\end{array}\right) \geq 0
\end{aligned}
$$

where $\Gamma_{l} \in \mathbb{C}^{(n-l+1) \times \times(n-1+1) \bullet}$ is the matrix defined as 


$$
\Gamma_{l}=\left(\begin{array}{cccc}
x I_{3} & & & 0 \\
I_{0} & \ddots & & \\
& \ddots & & \\
0 & & I_{0} & x I_{0}
\end{array}\right)
$$

with the resolvent

$$
\Gamma_{l}(z)=\left(z I_{(n-1+1),}-\Gamma_{l}\right)^{-1}
$$

and matrices $A^{(l)}, C^{(l)} \in \mathbb{C}^{(n-l+1) \times \times m}$ and $K^{(l)}, K_{p}^{(l)} \in \mathbb{C}^{(n-l+1) \bullet \times(n-l+1) s}$ are defined after data $a_{i}^{(l)}, c_{i}^{(l)} \in \mathbb{C}^{\Delta \times m}, \gamma_{i}^{(l)} \in \mathbb{C}^{\rho \times \rho}(i=0, \ldots, n-l)$ similarly to $(2.9),(2.12)$ and (2.13), i.e.

$$
\begin{aligned}
A^{(l)}= & \left(\begin{array}{c}
a_{0}^{(l)} \\
\vdots \\
a_{n-l}^{(i)}
\end{array}\right), \quad C^{(l)}=\left(\begin{array}{c}
c_{0}^{(l)} \\
\vdots \\
c_{n-l}^{(i)}
\end{array}\right) \\
K^{(l)}= & \left.\left(\begin{array}{ccc}
m i n(r, n-j-1) \\
\sum_{i=0}^{m} & \left(a_{r-j}^{(l)} c_{j+i+1}^{(l)}-c_{r-j}^{(l)} a_{j+i+1}^{(l)}\right)
\end{array}\right)\right)_{r, j=0}^{n-l} \\
& +\left(\begin{array}{cccc}
0 & \ldots & 0 & \gamma_{0}^{(l)} \\
\vdots & & \vdots \\
\gamma_{0}^{(l)} & \gamma_{1}^{(l)} & \ldots & \gamma_{n-l}^{(l)}
\end{array}\right) \\
K_{p}^{(l)}= & \Gamma_{l} K^{(l)}+A^{(l)} C^{(l) *} .
\end{aligned}
$$

In particular, $\left\{p_{l}, q_{l}\right\}$ satisfy the inequalities

$$
\begin{gathered}
\left(\begin{array}{cc}
k_{l} . & (z-x)^{-1}\left(a_{0}^{(l)},-c_{0}^{(l)}\right)\left(\begin{array}{l}
p_{l}(z) \\
q_{l}(z)
\end{array}\right) \\
* & \frac{q_{l}(z)^{*} p_{l}(z)-p_{l}(z)^{*} q_{l}(z)}{z-\bar{z}}
\end{array}\right) \geq 0 \\
\left(\begin{array}{cc}
k_{p l} & (z-x)^{-1}\left(z a_{0}^{(l)},-x c_{0}^{(l)}\right)\left(\begin{array}{l}
p_{l}(z) \\
q_{l}(z)
\end{array}\right) \\
* & \frac{z q_{l}(z)^{*} p_{l}(z)-\bar{z} p_{l}(z)^{*} q_{l}(z)}{z-\bar{z}}
\end{array}\right) \geq 0
\end{gathered}
$$

where

$$
k_{l}=a_{0}^{(l)} c_{1}^{(l) *}-c_{0}^{(l)} a_{1}^{(l) *} \quad \text { and } \quad k_{p l}=x k_{l}-a_{0}^{(l)} c_{0}^{(l) *} .
$$

Taking into account a possible singularity of $k_{l}, k_{p l}$ we introduce orthogonal projections $P_{\text {Ker } k_{1}}$ and $P_{\text {Ker } k_{p},}$ on their kernels and set

$$
Q_{l}=I_{0}-P_{\text {Ker } k_{l}} \quad \text { and } \quad Q_{p l}=I_{0}-P_{\text {Ker } k_{p l}}
$$


Since the transformations $k_{l}: \operatorname{Ran} k_{l} \rightarrow \operatorname{Ran} k_{l}$ and $k_{p l}: \operatorname{Ran} k_{p l} \rightarrow \operatorname{Ran} k_{p l}$ are one-toone, the pseudoinverse operators

$$
\begin{aligned}
& k_{l}^{[-1]} f= \begin{cases}\left(\left.Q_{l} k_{l}\right|_{\operatorname{Ran} k_{l}}\right)^{-1} f & \text { for } f \in \operatorname{Ran} k_{l} \\
0 & \text { for } f \in \operatorname{Ker} k_{l}\end{cases} \\
& k_{p l}^{[-1]} f= \begin{cases}\left(\left.Q_{p l} k_{p l}\right|_{\operatorname{Ran} k_{p l}}\right)^{-1} f & \text { for } f \in \operatorname{Ran} k_{p l} \\
0 & \text { for } f \in \operatorname{Ker} k_{p l}\end{cases}
\end{aligned}
$$

are well defined on $\mathbb{C}^{*}$ and

$$
k_{l} k_{l}^{[-1]}=k_{l}^{[-1]} k_{l}=Q_{l} \quad \text { and } \quad k_{p l} k_{p l}^{[-1]}=k_{p l}^{[-1]} k_{p l}=Q_{p l}
$$

Lemma 4.1. A non-degenerate pair $\left\{p_{l}, q_{l}\right\}$ of $\mathbb{C}^{m \times m}$-valued functions meromorphic in $\mathbb{C} \backslash \mathbb{R}_{+}$satisfies the system of matrix inequalities (4.8), (4.9) if and only if it admits a representation

$$
\left(\begin{array}{l}
p_{l}(z) \\
q_{l}(z)
\end{array}\right)=\theta_{l}(z)\left(\begin{array}{l}
p_{l+1}(z) \\
q_{l+1}(z)
\end{array}\right)
$$

where

$$
\Theta_{l}(z)=\left\{I+(z-x)^{-1}\left(\begin{array}{c}
c_{0}^{(l)} \\
a_{0}^{(l)^{*}}
\end{array}\right) k_{l}^{[-1]}\left(a_{0}^{(l)},-c_{0}^{(l)}\right)\right\}\left(\begin{array}{cc}
I I & 0 \\
a_{0}^{(l)} k_{p l}^{[-1]} a_{0}^{(l)} & I
\end{array}\right)
$$

and $\left\{p_{t+1}, q_{t+1}\right\}$ is some Stieltjes pair such that

$$
P_{\text {Ker k } t} a_{0}^{(l)} p_{l+1}(z) \equiv 0 \text { and } \quad P_{\text {Ker } k_{l}} c_{0}^{(l)} q_{l+1}(z) \equiv 0 .
$$

Proof. In the proof the index $l$ will be omitted. Let a pair $\left\{p_{l}, q_{l}\right\}$ satisfy the inequalities (4.8), (4.9). According to a lemma about a non-negative block matrix [13] the inequality (4.8) is equivalent to the system

$$
\begin{gathered}
P_{\text {Ker } k}\left(a_{0},-c_{0}\right)\left(\begin{array}{c}
p(z) \\
q(z)
\end{array}\right) \equiv 0 \\
\left(p(z)^{*}, q(z)^{*}\right)\left\{\frac{J}{z-\bar{z}}-|z-x|^{-2}\left(\begin{array}{c}
a_{0}^{*} \\
-c_{0}^{*}
\end{array}\right) k^{[-1]}\left(a_{0},-c_{0}\right)\right\}\left(\begin{array}{c}
p(z) \\
q(z)
\end{array}\right) \geq 0 .
\end{gathered}
$$

Using (4.15) and Lemma 3.4 we obtain

$$
\Theta(z) J \Theta(z)^{*}-J=i(z-\bar{z})|z-x|^{-2}\left(\begin{array}{c}
c_{0}^{*} \\
a_{0}^{*}
\end{array}\right) k^{[-1]}\left(c_{0}, a_{0}\right) .
$$

Hence the function $\theta$ is $J$-unitary on the real axis, $\theta^{-1}(z)=J \Theta(\bar{z})^{*} J$, and, in view of (4.19),

$$
\begin{aligned}
\Theta(z)^{-*} J \Theta^{-1}(z)-J & =J\left(\Theta(\bar{z}) J \Theta(\bar{z})^{*}-J\right) J \\
& =i(z-\bar{z})|z-x|^{-i}\left(\begin{array}{c}
a_{0}^{*} \\
-c_{0}^{*}
\end{array}\right) k^{[-1]}\left(a_{0},-c_{0}\right) .
\end{aligned}
$$


Using (4.20) we can rewrite (4.18) as

$$
\left(p(z)^{*}, q(z)^{*}\right) \frac{\Theta(z)^{-*} J \Theta(z)^{-1}}{z-\bar{z}}\left(\begin{array}{l}
p(z) \\
q(z)
\end{array}\right) \geq 0 .
$$

It follows from the last inequality that $\{p, q\}$ admits a representation (4.14) with some non-degenerate (since $\operatorname{det} \theta(z) \not \equiv 0)$ pair $\left\{p_{1}, q_{1}\right\}$ such that

$$
\frac{q_{1}(z)^{*} p_{1}(z)-p_{1}(z)^{*} q_{1}(z)}{z-\bar{z}} \geq 0
$$

It remains to show that the pair $\left\{p_{1}, q_{1}\right\}$ belongs to $\overline{\mathbf{S}}_{m}$ and satisfies conditions (4.16). According to the cited lemma about a non-negative block matrix the inequality (4.9) is equivalent to the system

$$
\begin{gathered}
P_{\text {Ker } k_{p}}\left(z a_{0},-x c_{0}\right)\left(\begin{array}{c}
p(z) \\
q(z)
\end{array}\right) \equiv 0 \\
\left(\bar{z} p(z)^{*}, q(z)^{*}\right)\left\{\frac{J}{z-\bar{z}}-|z-x|^{-2}\left(\begin{array}{c}
a_{0}^{*} \\
-x c_{0}^{*}
\end{array}\right) k_{p}^{[-1]}\left(a_{0},-x c_{0}\right)\right\}\left(\begin{array}{c}
z p(z) \\
q(z)
\end{array}\right) \geq 0 .
\end{gathered}
$$

Using (4.10)-(4.13) and (4.15) we obtain

$$
\begin{aligned}
\left(a_{0},-c_{0}\right) \Theta(z) & =\left(a_{0},-c_{0}\right)\left(\begin{array}{cc}
I & 0 \\
a_{0}^{*} k_{p}^{[-1]} a_{0} & I
\end{array}\right) \\
& =\left(\left(I-\left(k_{p}-x k\right) k_{p}^{[-1]}\right) a_{0},-c_{0}\right) \\
& =\left(\left(P_{\text {Ker } k_{p}}+x k k_{p}^{[-1]}\right) a_{0},-c_{0}\right)
\end{aligned}
$$

and

$$
\begin{aligned}
\left(z a_{0},-x c_{0}\right) \Theta(z)= & \left(\left((z-x) a_{0}, 0\right)+x\left(a_{0},-c_{0}\right)\right) \Theta(z) \\
= & \left((z-x) a_{0}, 0\right)+x P_{\text {Ker } k}\left(P_{\text {Ker } k_{p}} a_{0},-c_{0}\right) \\
& +k_{p}\left(\left(k^{[-1]} \dot{P}_{\text {Ker } k_{p}}+x Q k_{p}^{[-1]}\right) a_{0},-k^{[-1]} c_{0}\right) .
\end{aligned}
$$

Substitution of (4.14), (4.24) into (4.17) gives

$$
P_{\text {Ker } k}\left(P_{\text {Ker } k_{p} a_{0},-c_{0}}\right)\left(\begin{array}{l}
p_{1}(z) \\
q_{1}(z)
\end{array}\right) \equiv 0 .
$$

Substituting (4.14) into (4.22) and taking into account (4.25), (4.26) we obtain the identity $P_{\text {Ker } k_{p}} a_{0} p_{1}(z) \equiv 0$, which both with (4.26) implies $P_{\text {Ker } k} c_{0} q_{1}(z) \equiv 0$. Using two last identities and substituting (4.14) into (4.23) we obtain the inequality

$$
\left(\bar{z} p_{1}(z)^{*}, q_{1}(z)^{*}\right) \frac{J}{z-\bar{z}}\left(\begin{array}{c}
z p_{1}(z) \\
q_{1}(z)
\end{array}\right) \geq 0
$$

which both with (4.21) means that $\left\{p_{1}, q_{1}\right\} \in \overline{\mathbf{S}}_{m}$.

The necessary part of the lemma can be obtained by a direct substitution of (4.14) into (4.8), (4.9) and use of (4.16) 
The solution $\left\{p_{l}, q_{l}\right\}$ of the system (4.8), (4.9) (i.e. a Stieltjes pair of the form (4.14) ) to be a solution of the "full" system (4.1), (4.2), its parameter $\left\{p_{1+1}, q_{1+1}\right\}$ in (4.14) has to satisfy some additional conditions which are given in the following lemma

Le'mma 4.2. Let $\left\{p_{l+1}, q_{l+1}\right\}$ be a non-degenerate pair of $\mathbb{C}^{m \times m}$-valued functions meromorphic in $\boldsymbol{C} \backslash \mathbb{R}_{+}$which satisfy (4.16). Let $\theta_{\text {, be a }} \boldsymbol{C}^{2 m \times 2 m}$-valued function defined by (4.15) and let $\left\{p_{l}, q_{l}\right\}$ be a pair defined by the linear transformation (4.14). Then $\left\{p_{l}, q_{l}\right\}$ satisfies (4.1), (4.2) if and only if $\left\{p_{l+1}, q_{l+1}\right\}$ satisfies the system

$$
R^{(l+1)} \geq 0, \quad R_{p}^{(1+1)} \geq 0
$$

where matrices $R^{(1+1)}$ ans $R_{p}^{(1+1)}$ are defined by (4.1) and (4.2), and $(j \geq 0)$

$$
\begin{aligned}
a_{j}^{(l+1)} & = \begin{cases}a_{j+1}^{(l)}-\left(x c_{j+2}^{(l)} a_{0}^{(l)^{\bullet}}-x a_{j+2}^{(l)} c_{0}^{(l)^{\bullet}}+c_{j+1}^{(l)} a_{0}^{*}\right) k_{p l}^{(-1)} a_{0}^{(l)} & \text { if } j<n-l \\
a_{j+1}^{(l)}-\left(x \gamma_{0}^{(l)}+c_{j+1}^{(l)} a_{0}^{(l)^{*}}\right) k_{p l}^{(-1)} a_{0}^{(l)} & \text { if } j=n-l\end{cases} \\
c_{j}^{(l+1)} & = \begin{cases}c_{j+1}^{(l)}-\left(c_{j+2}^{(l)} a_{0}^{(l)}-a_{j+2}^{(l)} c_{0}^{(l)}\right) k_{l}^{(-1]} c_{0}^{(l)} & \text { if } j<n-l \\
c_{j+1}^{(l)}-\gamma_{0}^{(l)} k_{l}^{(-1]} c_{0}^{(l)} & \text { if } j=n=l\end{cases} \\
\gamma_{j}^{(l+1)} & = \begin{cases}\gamma_{j+1}^{(l)}-\left(c_{j+2}^{(l)} a_{0}^{(l)}-a_{j+2}^{(l)} c_{0}^{(l)}\right) k_{l}^{(-1]} \gamma_{0}^{(l)} & \text { if } j<n-l \\
\gamma_{j+1}^{(l)}-\gamma_{0}^{(l)} k_{l}^{[-1]} \gamma_{0}^{(l)} & \text { if } j=n-l .\end{cases}
\end{aligned}
$$

Proof. In the proof the index $l$ will be omitted. Let

$$
K=\left(\begin{array}{cc}
k & B \\
B^{*} & \tilde{K}
\end{array}\right) \quad \text { and } \quad K_{p}=\left(\begin{array}{cc}
k_{p} & B_{p} \\
B_{p}^{*} & \tilde{K}_{p}
\end{array}\right)
$$

be block decompositions of information matrices $K$ and $K_{p}$. The non-negativity of $K$ ans $K_{p}$ implies $P_{\text {Ker } k} B=0$ and $P_{\text {Ker } k_{p}} B_{p}=0$ which in view of (4.10) can be rewritten as $B=Q B$ and $B_{p}=Q_{p} B_{p}$. Therefore, $K$ and $K_{p}$ admit the factorizations

$$
\begin{aligned}
& K=\left(\begin{array}{cc}
I_{s} & 0 \\
B^{*} k^{[-1]} & I_{n}
\end{array}\right)\left(\begin{array}{cc}
k & 0 \\
0 & \tilde{K}-B^{*} k^{[-1]} B
\end{array}\right)\left(\begin{array}{cc}
I_{0} & k^{[-1]} B \\
0 & I_{n s}
\end{array}\right) \\
& K_{p}=\left(\begin{array}{cc}
I_{s} & 0 \\
B_{p}^{*} k_{p}^{[-1]} & I_{n s}
\end{array}\right)\left(\begin{array}{cc}
k_{p} & 0 \\
0 & \tilde{K}_{p}-B_{p}^{*} k_{p}^{[-1]} B_{p}
\end{array}\right)\left(\begin{array}{cc}
I_{s} & k_{p}^{[-1]} B_{p} \\
0 & I_{n s}
\end{array}\right) .
\end{aligned}
$$

Substituting (4.14), (4.15) into (4.1), (4.2) and multiplying matrices from (4.1),(4.2) on the left by matrices

$$
L=\left(\begin{array}{ccc}
I & 0 & 0 \\
-B^{*} k^{[-1]} & I & 0 \\
0 & 0 & I_{m}
\end{array}\right) \quad \text { and } \quad L_{p}=\left(\begin{array}{ccc}
I & 0 & 0 \\
-B_{p}^{*} k_{p}^{[-1]} & I & 0 \\
0 & 0 & I_{m}
\end{array}\right)
$$

and on the right by by $L^{*}$ and $L_{p}^{*}$, respectively, we obtain in view of factorizations (4.32) and (4.33) the inequalities

$$
\left(\begin{array}{ccc}
k & 0 & \psi(z) \\
0 & \tilde{K}-B^{*} k^{[-1]} B & \Psi(z) \\
\psi(z)^{*} & \Psi(z)^{*} & \frac{q_{1}(z)^{*} p_{1}(z)-p_{1}(z)^{*} q_{1}(z)}{z-\bar{z}}+\psi(z)^{*} k^{[-1]} \psi(z)
\end{array}\right) \geq 0
$$


and

$$
\left(\begin{array}{ccc}
k_{p} & 0 & \psi_{p}(z) \\
0 & \tilde{K}_{p}-B_{p}^{*} k_{p}^{[-1]} B_{p} & \Psi_{p}(z) \\
\psi_{p}(z)^{*} & \Psi_{p}(z)^{*} & \frac{z q_{1}(z)^{*} p_{1}(z)-\bar{z} p_{1}(z)^{*} q_{1}(z)}{z-\bar{z}}+\psi_{p}(z)^{*} k_{p}^{[-1]} \psi_{p}(z)
\end{array}\right) \geq 0
$$

where

$$
\begin{aligned}
& \psi(z)=(z-x)^{-1}\left(a_{0},-c_{0}\right) \Theta(z)\left(\begin{array}{c}
p_{1}(z) \\
q_{1}(z)
\end{array}\right) \\
& \psi_{p}(z)=(z-x)^{-1}\left(z a_{0},-x c_{0}\right) \Theta(z)\left(\begin{array}{c}
p_{1}(z) \\
q_{1}(z)
\end{array}\right) \\
& \Psi(z)=-B k^{[-1]} \psi(z)+\left(0, I_{n s}\right) \Gamma(z)(A,-C) \Theta(z)\left(\begin{array}{c}
p_{1}(z) \\
q_{1}(z)
\end{array}\right) \\
& \dot{\Psi}_{p}(z)=-B_{p} k_{p}^{[-1]} \psi_{p}(z)+\left(0, I_{n o}\right) \Gamma(z)(z A,-\Gamma C) \Theta(z)\left(\begin{array}{c}
p_{1}(z) \\
q_{1}(z)
\end{array}\right) .
\end{aligned}
$$

Substituting (4.24) and (4.25) into (4.36) and (4.37), respectively, and taking into account (4.16) we obtain

$$
\begin{aligned}
\psi(z) & =(z-x)^{-1}\left(x k k_{p}^{[-1]} a_{0} p_{1}(z)-c_{0} q_{1}(z)\right) \\
\psi_{p}(z) & =a_{0} p_{1}(z)+(z-x)^{-1} k_{p}\left(x Q k_{p}^{[-1]} a_{0} p_{1}(z)-k^{[-1]} c_{0} q_{1}(z)\right)
\end{aligned}
$$

To transform (4.38) and (4.39) we consider block decompositions

$$
A=\left(\begin{array}{c}
a_{0} \\
\tilde{A}
\end{array}\right), C=\left(\begin{array}{c}
c_{0} \\
\tilde{C}
\end{array}\right), \Gamma=\left(\begin{array}{cc}
x I_{2} & 0 \\
G & \Gamma_{1}
\end{array}\right), \Gamma(z)=\left(\begin{array}{cc}
(z-x)^{-1} I_{0} & 0 \\
G(z) & \Gamma_{1}(z)
\end{array}\right)
$$

where

$$
\tilde{A}=\left(\begin{array}{c}
a_{1} \\
a_{2} \\
\vdots \\
a_{n}
\end{array}\right), \tilde{C}=\left(\begin{array}{c}
c_{1} \\
c_{2} \\
\vdots \\
c_{n}
\end{array}\right), G=\left(\begin{array}{c}
I_{0} \\
0 \\
\vdots \\
0
\end{array}\right), G(z)=\left(\begin{array}{c}
(z-x)^{-2} I_{d} \\
(z-x)^{-3} I_{z} \\
\vdots \\
(z-x)^{-n-1} I_{0}
\end{array}\right)
$$

and $\Gamma_{1}\left(=\Gamma_{l+1}\right)$ and $\Gamma_{1}(z)\left(=\Gamma_{t+1}(z)\right)$ are matrices defined according to (4.3) and (4.4), respectively. Substituting decompositions (4.31), (4.42) into (4.7) and comparing in the obtained identity non-diagonal blocks we have

$$
\begin{aligned}
B_{p}-x B & =a_{0} \tilde{C}^{*} \\
B_{p}^{*}-\Gamma_{1} B^{*}-G k & =\tilde{A} c_{0}^{*} \\
\tilde{K}_{p}-\Gamma_{1} \tilde{K}-G B & =\tilde{A} \tilde{C}^{*} .
\end{aligned}
$$


Substituting (4.15), (4.40), (4.41) into (4.38), (4.39) and using (4.44)-(4.46) we obtain

$$
\begin{gathered}
\Psi(z)=\Gamma_{1}(z)\left(\tilde{A}-B_{p}^{*} k_{p}^{[-1]} a_{0},-\tilde{C}+B^{*} k^{[-1]} c_{0}\right)\left(\begin{array}{c}
p_{1}(z) \\
q_{1}(z)
\end{array}\right) . \\
\Psi_{p}(z)=\Gamma_{1}(z)\left(\tilde{A}-B_{p}^{*} k_{p}^{[-1]} a_{0},-\Gamma_{1}\left(\tilde{C}-B^{*} k^{[-1]} c_{0}\right)\right)\left(\begin{array}{c}
z p_{1}(z) \\
q_{1}(z)
\end{array}\right) .
\end{gathered}
$$

It follows from (4.6), (4.7), (4.31) that

$$
\begin{aligned}
B & =\left(a_{0} c_{2}^{*}-c_{0} a_{2}^{*}, \ldots, a_{0} c_{n}^{*}-c_{0} a_{n}^{*}, \gamma_{0}\right) \\
B_{p} & =x B+a_{0}\left(c_{1}^{*}, \ldots, c_{n}^{*}\right) .
\end{aligned}
$$

Comparing (4.49) and (4.50) with (4.28) and (4.29), respectively, we obtain

$$
A^{(1)}=\tilde{A}-B_{p}^{*} k_{p}^{[-1]} a_{0} \quad \text { and } \quad C^{(1)}=\tilde{C}-B^{*} k^{[-1]} c_{0}
$$

and therefore (4.47), (4.48) can be rewritten as

$$
\begin{gathered}
\Psi(z)=\Gamma_{1}(z)\left(A^{(1)},-C^{(1)}\right)\left(\begin{array}{c}
p_{1}(z) \\
q_{1}(z)
\end{array}\right) . \\
\Psi_{p}(z)=\Gamma_{1}(z)\left(A^{(1)},-\Gamma_{1} C^{(1)}\right)\left(\begin{array}{c}
z p_{1}(z) \\
q_{1}(z)
\end{array}\right) .
\end{gathered}
$$

It follows from (4.6), (4.28)-(4.30), (4.49), (4.50) that

$$
\dot{K}^{(1)}=\tilde{K}-B k^{[-1]} B^{*} \text {. }
$$

Moreover, we show that

$$
K_{p}^{(1)}=\tilde{K}_{p}-B_{p} k_{p}^{(-1)} B_{p}^{*}
$$

Really, substituting (4.51), (4.54) into (4.7) and using (4.44)-(4.46) we have

$$
\begin{aligned}
K_{p}^{(1)}= & \Gamma_{1} \tilde{K}-\Gamma_{1} B^{*} k^{[-1]} B+\tilde{A} \tilde{C}^{*}-\tilde{A} c_{0}^{*} k^{[-1]} B \\
& -B_{p}^{*} k_{p}^{[-1]} a_{0} \tilde{C}^{*}+B_{p}^{*} k_{p}^{[-1]} a_{0} c_{0}^{*} k^{[-1]} B \\
= & \Gamma_{1} \tilde{K}-\Gamma_{1} B^{*} k^{[-1]} B+\tilde{A} \tilde{C}^{*}-\left(B_{p}^{*}-\Gamma_{1} B^{*}-G k\right) k^{[-1]} B \\
& -B_{p}^{*} k_{p}^{[-1]}\left(B_{p}-x B\right)+B_{p}^{*} k^{[-1]} B-x B_{p}^{*} k_{p}^{[-1]} B \\
= & \Gamma_{1} \tilde{K}+\tilde{A} \tilde{C}^{*}-G B-B_{p}^{*} k_{p}^{[-1]} B_{p} \\
= & \tilde{K}_{p}-B_{p}^{*} k^{[-1]} B_{p} .
\end{aligned}
$$

To conclude the proof we note that according to the lemma about a non-negative block matrix the system of inequalities $(4: 34),(4.35)$ is equivalent to the system

$$
\begin{aligned}
\left(\begin{array}{cc}
\tilde{K}-B^{*} k^{[-1]} B & \Psi(z) \\
\Psi(z)^{*} & \frac{q_{1}(z)^{*} p_{1}(z)-p_{1}(z)^{*} q_{1}(z)}{z-\bar{z}}
\end{array}\right) & \geq 0 \\
\left(\begin{array}{cc}
\tilde{K}_{p}-B_{p}^{*} k_{p}^{[-1]} B_{p} & \Psi_{p}(z) \\
\Psi_{p}(z)^{*} & \frac{z q_{1}(z)^{*} p_{1}(z)-\bar{z} p_{1}(z)^{*} q_{1}(z)}{z-\bar{z}}
\end{array}\right) & \geq 0
\end{aligned}
$$

which in view of (4.1), (4.2) and (4.52)-(4.55) coincides with (4.27) 
In view of Lemma 4.2 the conditions (4.27) on the parameter $\left\{p_{t+1}, q_{t+1}\right\}$ preserve the structure of inequalities (4.1), (4.2) and, thus, the process is continuable. Under the process we obtain a sequence of resolvent matrices $\theta_{l}$, matrices $a_{j}^{(l)}, c_{j}^{(l)} \in \mathbb{C}^{\bullet \times m}$, matrices $\gamma_{j}^{(l)}, k_{l}, k_{p l} \in \mathbb{C}^{\circ x_{0}}$ and parameters $\left\{p_{l}, q_{l}\right\} \in \overline{\mathbf{S}}_{m}$ for $l=0, \ldots, n$ and $j=$ $0, \ldots, n-l$ (recursive formulas for $a_{j}^{(l)}, c_{j}^{(l)}, \gamma_{j}^{(l)}$ and $\left\{p_{l}, q_{l}\right\}$ are given correspondingly by (4.28) - (4.30) and (4.14); in formula (4.10) $k_{n}=\gamma_{0}^{(n)}, k_{p n}=x \gamma_{0}^{(n)}+a_{0}^{(n)} c_{0}^{(n)^{*}}$ for $l=n$ ).

We notice that in conditions (4.16) and in the formula (4.15) for the resolvent matrix


properties of the last ones.

Lemma 4.3. Let $a_{j}^{(l)}, c_{j}^{(l)} \in \mathbb{C}^{a \times m}$ and $\gamma_{j}^{(l)}, k_{l}, k_{p l} \in \mathbb{C}^{\circ \times}$ be matrices defined in (4.28) - (4.90) and (4.9), respectively, and let $\left\{p_{l}, q_{l}\right\}$ be Stieltjes pairs defined by (4.14). Then

(i) $P_{\text {Ker } k, 1} a_{0}^{(l)} c_{j}^{(r)^{*}}=0$ and $P_{\text {Ker } k_{1}} c_{0}^{(l)} a_{j}^{(r)^{*}}=0(r>l ; j=0, \ldots, n-r)$

(ii) $P_{\text {Ker } k, 1} a_{0}^{(l)} p_{r}(z) \equiv 0$ and $P_{\text {Ker } k_{i}} c_{0}^{(l)} q_{r}(z) \equiv 0(r>l)$.

Proof. At first we prove (4.56) for $r=1+1$. Using (4.43), (4.44), (4.51) we obtain

$$
\begin{aligned}
P_{\text {Ker } k_{p l}} a_{0}^{(l)}\left(c_{0}^{(l+1)}, \ldots, c_{n-l-1}^{(l+1)}\right) & =P_{\text {Ker } k_{p 1}} a_{0}^{(l)} C^{(l+1)^{\bullet}} \\
& =P_{\text {Ker } k_{p l}} a_{0}^{(l)}\left(\tilde{C}^{*}-c_{0}^{(l)} k_{l}^{[-1]} B\right) \\
& =P_{\text {Ker } k_{p l}}\left(B_{p}-x B-\left(k_{p l}-x k_{l}\right) k_{l}^{[-1]} B\right) \\
& =x P_{\text {Ker } k_{p l}}(-B+Q B) \\
& =0 .
\end{aligned}
$$

By the same way we get $R_{\text {ker } k_{l}} c_{0}^{(l)} a_{j}^{(l+1)^{*}}=0$ for $j=0, \ldots, n-l-1$. To obtain (4.56) for all $r$ it sufficies to note that, in view of recursive formulas (4.28), (4.29),

$$
\begin{aligned}
& \operatorname{Ran} a_{j}^{(r)} \subset \operatorname{Lin}\left(\operatorname{Ran} a_{i}^{(l)}\right)_{i=1, \ldots, n-l} \\
& \operatorname{Ran} c_{j}^{(r)} \subset \operatorname{Lin}\left(\operatorname{Ran} c_{i}^{(l)}\right)_{i=1, \ldots, n-l}
\end{aligned}
$$

for all $r>l$ and $j=0, \ldots, n-r$, where Lin stands for linear span and Ran $F$ denotes the left image of the $s \times m$ matrix $F: \operatorname{Ran} F=\left\{f \in \mathbb{C}^{m}: f=g F\right.$ for some $\left.g \in \mathbb{C}^{\prime}\right\}$.

To prove (4.57) we use induction. The assertions of the lemma are valid for $r=l+1$ in view of (4.16). Let (4.56) hold for $r=t$. Then, (4.14) implies

$$
P_{\text {Ker } k_{, 1}} a_{0}^{(l)} p_{t}(z)=P_{\text {Ker k } p l}\left(a_{0}^{(l)}, 0\right) \theta_{t}(z)\left(\begin{array}{c}
p_{t+1}(z) \\
q_{t+1}(z)
\end{array}\right) .
$$

Substituting into (4.60) the expression (4.15) for $\theta_{t}$ and taking into account (4.56) we obtain

$$
P_{\text {Ker } k_{p l}} a_{0}^{(l)} p_{t}(z)=P_{\text {Ker } k_{p l}} a_{0}^{(l)} p_{t+1}(z) \equiv 0 .
$$

The second identity in (4.57) can be obtained by the same way 
Lemma 4.4. Let $\mathcal{M}, \mathcal{E}$ be subspaces in $\mathbb{C}^{m}$ defined by

$$
\mathcal{M}=\operatorname{Lin}\left(\operatorname{Ran} P_{\text {Ker } k_{1},} a_{0}^{(l)}\right)_{l=0}^{n} \quad \text { and } \quad \mathcal{E}=\operatorname{Lin}\left(\operatorname{Ran} P_{\text {Ker } k_{l}} c_{0}^{(l)}\right)_{l=0}^{n} \text {. }
$$

Then,

(i) $\mathcal{M} \perp \mathcal{E}$

(ii) $\operatorname{dim} \mathcal{M}=\operatorname{rank} P_{\text {Ker k, }} A$ and $\operatorname{dim} \mathcal{E}=\operatorname{rank} P_{\text {Ker } k} C$

where $A, C$ are matrices defined in (2.19).

Proof. Relation (4.62) follows from (4.56) and the equality

$$
P_{\text {Ker } k_{p} t} a_{0}^{(l)} c_{0}^{(l)} P_{\text {Ker } k_{p 1}}=P_{\text {Ker } k_{11}}\left(k_{p l}-x k_{1}\right) P_{\text {Ker } k_{1}}=0 .
$$

To prove relation (4.63) we note that, for any $(n-l+1) s \times(n-l+1)$ non-degenerate matrix $T$,

$$
\operatorname{rank} P_{\text {Ker } K^{(1)}} C^{(l)}=\operatorname{rank} P_{\text {Ker } T K^{(1)} T} \cdot T C^{(l)}
$$

$\left(K^{(l)}\right.$ is the informative matrix defined by (4.3)). Turning to the block decomposotion (4.31) of $K^{(l)}$ we put $T=\left(\begin{array}{c}I \\ -B \cdot k_{l}^{(-1)} \\ 0\end{array}\right)$. It follows from (4.32), (4.51) and (4.54) that

$$
S=T K^{(l)} T^{*}=\left(\begin{array}{cc}
k_{l} & 0 \\
0 & K^{(l+1)}
\end{array}\right) \quad \text { and } \quad T C^{(l)}=\left(\begin{array}{c}
c_{0}^{(l)} \\
C^{(l+1)}
\end{array}\right)
$$

Substituting these equalities into (4.64) we obtain

$$
\begin{aligned}
\operatorname{rank} P_{\text {Ker } K^{(l)}} C^{(l)} & =\operatorname{rank} P_{\text {Ker } S}\left(\begin{array}{c}
c_{0}^{(l)} \\
C^{(l+1)}
\end{array}\right) \\
& =\operatorname{rank} P_{\text {Ker } k_{1}} c_{0}^{(l)}+\operatorname{rank} P_{\text {Ker } K^{(l+1)}} C^{(l+1)}
\end{aligned}
$$

Applying induction we receive $\operatorname{rank} P_{\text {Ker } K} C=\sum_{l=0}^{n}$ rank $P_{\text {Ker } k_{l}} c_{0}^{(l)}=\operatorname{dim} \mathcal{E}$. The first equality in (ii) can be obtained by the same way

Lemma 4.5 (see $[9]$ ). Let $\{p, q\} \in \overline{\mathbf{S}}_{m}$. pairs

(i) If $\left[0, I_{\nu}\right] p(z) \equiv 0$, then there exists a pair $\left\{p_{1}(z), q_{1}(z)\right\} \in \overline{\mathbf{S}}_{m-\nu}$ such that the

$$
\{p(z), q(z)\} \quad \text { and } \quad\left\{\left(\begin{array}{cc}
p_{1}(z) & 0 \\
0 & 0_{v}
\end{array}\right),\left(\begin{array}{cc}
q_{1}(z) & 0 \\
0 & I_{\nu}
\end{array}\right)\right\}
$$

are equivalent.

(ii) If $\left[0, I_{\nu}\right] q(z) \equiv 0$, then there exists a pair $\left\{p_{2}, q_{2}\right\} \in \overline{\mathbf{S}}_{m-\mu}$ such that the pairs

$$
\{p(z), q(z)\} \quad \text { and } \quad\left\{\left(\begin{array}{cc}
p_{2}(z) & 0 \\
0 & I_{\mu}
\end{array}\right),\left(\begin{array}{cc}
q_{2}(z) & 0 \\
0 & 0_{\mu}
\end{array}\right)\right\}
$$

are equivalent.

The following theorem.is a degenerate analogue of Theorem 3.10 and gives a description of all the solutions to the problem (ISP). 
Theorem 4.6. Let $A, C, K \geq 0, K_{p} \geq 0$ be matrices defined by (2.9), (2:12), (2.19) and let $\theta_{l}(l=0, \ldots, n)$ be $C^{2 m \times 2 m}$-valued functions defined by (4.15). Then the linear transformation (9.18) with the resolvent matrix

$$
\Theta(z)=\prod_{l=0}^{n} \theta_{l}(z)
$$

gives a parametrization of all solutions to the problem (ISP) (or, equivalently; all the solutions to the system (S.17)) when the parameter $\{\tilde{p}, \tilde{q}\}$ varies in $\overline{\mathbf{S}}_{m}$ and is of the form

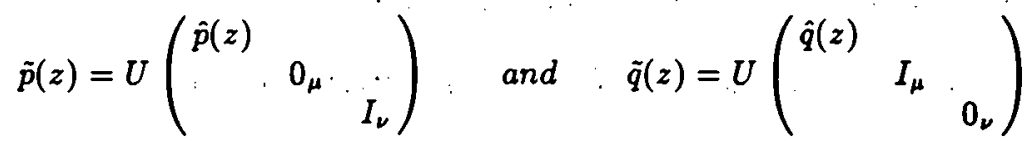

with unitary matrix $U \in \mathscr{C}^{m \times m}$ depending only on the interpolation data and Stieltjes pair $\{\hat{p}(z), \hat{q}(z)\} \in \overline{\mathbf{S}}_{m-\mu-\nu}$, where

$$
\mu=\operatorname{rank} P_{\text {Ker } K_{p}} A \quad \text {, and . } \nu=\operatorname{rank} P_{\text {Ker } K} C .
$$

Proof. Setting $\dot{a}_{j}^{(0)}=a_{j}, c_{j}^{(0)}=c_{j}, \gamma_{j}^{(0)}=\gamma_{j}(j \doteq 0, \ldots, n)$ we apply $n+1$ times Lemmas 4.1 and 4.2 to the system (3.17). As a result we obtain that every solution $\{p, q\}$ of the system (3.17) admits a representation (3.18) with the resolvent matrix $\theta$ defined by (4.66) and parameter $\{\tilde{p}, \tilde{q}\}\left(=\left\{p_{n+1}, q_{n+1}\right\}\right)$ in $\overline{\mathbf{S}}_{m}$. In view of (4.57)

$$
P_{\mathcal{M}} \tilde{p}(z) \equiv 0 \ldots \text { and } \quad P_{\mathcal{E}} q(z) \equiv 0
$$

where $P_{\mathcal{M}}$ and $P_{\mathcal{E}}$ are orthogonal projections on subspaces $\mathcal{M}$ and $\mathcal{E}$, respectively, defined by (4.61). To represent (4.69) in the form (4.67) we note that in view of (4.62) there exists a unitary matrix $U \in \mathbb{C}^{m \times m}$ such that

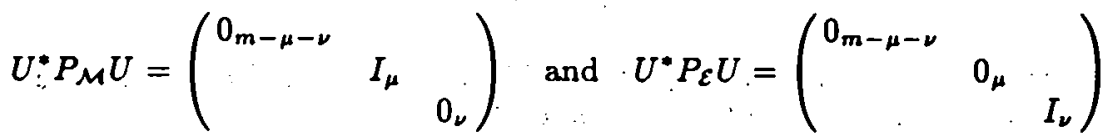

where

$$
\mu=\operatorname{dim} \mathcal{M} \quad \text { and } \quad \nu=\operatorname{dim} \mathcal{E} .
$$

Substituting (4.70) into (4.69) and applying Lemma 4.5 to the Stieltjes pair $\left\{U^{*} \tilde{p}, U^{*} \tilde{q}\right\}$ we obtain equivalence of the pairs

$$
\therefore\{\tilde{p}(z), \tilde{q}(z)\} \quad \text { and } \quad\left\{U\left(\begin{array}{ccc}
\hat{p}(z) & & \\
& 0_{\mu} & \\
& & I_{\nu}
\end{array}\right), U\left(\begin{array}{lll}
\hat{q}(z) & & \\
& I_{\mu} & \\
& & 0_{\nu}
\end{array}\right)\right\} .
$$

Equalities (4.68) follow from (4.63) and (4.71).

To check that any pair $\{\tilde{p}, \tilde{q}\}$ of the form (4.67) (or, equivalently, satisfying the conditions (4.69)) under linear transformation leads to some solution $\{p, q\}$ to $(3.17)$ we use a multiplicative representation (4.66) of $\Theta$ and apply step by step Lemmas 4.1 and 4.2. Finally, since functions $\theta_{l}(l=0, \ldots, n)$ belong to $W$ (see (4.19)), the matrix $\theta$ of the form (4.66) belongs to $W$ as well' 
Corollary 4.7. The problem (ISP) is solvable if and only if the matrices $K$ and $K_{p}$ are both non-negative.

Proof. The necessity follows from Theorem 3.10. The sufficiency follows from Theorem 4.6 since the set of elements of the form (4.67) is not empty

Corollary 4.8. The problem (ISP) has a unique solution if and only if $\dot{\mu}+\nu=m$, where $\mu, \nu$ are numbers defined in (4.68).

To obtain the analogous description for the problem. (IS) we have to care of the non-degeneracy of the "denominator" in the linear fractional transformation (3.16).

Lemma 4.9. Let $\theta=\left(\theta_{i j}\right)$ be the block decomposition of $\theta$ defined by (4.66) into four $\widetilde{C}^{m \times m}$-valued functions and let $\{p, q\}$ be in $\overline{\mathbf{S}}_{m}$ and satisfy the conditions (4.69). Then

$$
\operatorname{det}\left(\theta_{21}(z) p(z)^{\prime}+\theta_{22}(z) q(z)\right) \not \equiv 0
$$

if and only if $\{p, q\}$ belongs to the subclass $\overline{\mathbf{S}}_{m}^{0}$ defined in (\$.15).

Proof. In view of (4.28) $\operatorname{Ran} A \doteq \operatorname{Lin}\left(\operatorname{Ran} a^{(l)}\right)_{l=0, \ldots, n}$, and therefore, $\{p, q\}$ belongs to $\overline{\mathbf{S}}_{m}^{0}$ if and only if

$$
\operatorname{det}\left(p(z)^{*}\left(\sum_{l=0}^{n} a_{0}^{(l)^{*}} a_{0}^{(l)}\right) p(z)+q(z)^{*} q(\dot{z})\right) \not \equiv 0
$$

Let $\{p, q\} \in \overline{\mathbf{S}}_{m}^{0}$. We introduce a pair

$$
\left(\begin{array}{l}
p_{0}(z) \\
q_{0}(z)
\end{array}\right)=\theta(z)\left(\begin{array}{l}
p(z) \\
q(z)
\end{array}\right)
$$

and show that $\operatorname{det} q_{0}(z) \not \equiv 0$. Indeed, suppose that the point $z \in \mathcal{C}_{+}$. and the non-zero vector $h \in \mathbb{C}^{\mathbf{m}}$ are such that

$$
q_{0}(z) \dot{h}=0
$$

Since

$$
h^{*}\left(p(z)^{*}, q(z)^{*}\right) \Theta(z)^{*} J \Theta(z)\left(\begin{array}{c}
p(z) \\
q(z)
\end{array}\right) h=\left(h^{*} p_{0}(z)^{*}, 0\right) J\left(\begin{array}{c}
p_{0}(z) h \\
0
\end{array}\right)=0
$$

then, in view of (4.66) and Lemma 3.2,

$$
\begin{aligned}
0 & \leq h^{*}\left(p(z)^{*}, q(z)^{*}\right) J\left(\begin{array}{c}
p(z) \\
q(z)
\end{array}\right) \\
& =h^{*}\left(p(z)^{*}, q(z)^{*}\right)\left(J-\theta(z)^{*} J \theta(z)\right)\left(\begin{array}{c}
p(z) \\
q(z)
\end{array}\right) h \\
& \leq h^{*}\left(p(z)^{*}, q(z)^{*}\right)\left(J-\theta_{l}(z)^{*} J \theta_{l}(z)\right)\left(\begin{array}{c}
p(z) \\
q(z)
\end{array}\right) h \quad: \quad(l=0, \ldots, n) .
\end{aligned}
$$

Substituting (4.15) into the last inequality and using (4.69) we obtain

$$
h^{*}\left(x p(z)^{*} a_{0}^{(l)^{*}} k_{p l}^{[-1]} k_{l}-q(z)^{*} c_{0}^{(l)^{*}}\right) k_{l}^{[-1]}\left(x k_{1} k_{p l}^{[-1]} a_{0}^{(l)} p(z)-c_{0}^{(l)} q(z)\right) h=0
$$


which is equivalent to

$$
k_{l}^{[-1]}\left(x k_{l} k_{p l}^{[-1]} a_{0}^{(l)} p(z)-c_{0}^{(l)} q(z)\right) h=0 \quad(l=0, \ldots, n) .
$$

Setting $\left\{p_{n}, q_{n}\right\}=\{p, q\}$ we apply the inverse Schur process using (4.14). In view of (4.69) and (4.74),

$$
\begin{aligned}
\left(\begin{array}{l}
p_{n-1}(z) \\
q_{n-1}(z)
\end{array}\right) h= & \left(\left(\begin{array}{cc}
a_{0}^{(n)^{\bullet}}{ }_{k_{p n}\left[a_{0}^{(n)}\right.}^{I} a^{(n)} & I
\end{array}\right)\right. \\
& \left.+\frac{1}{z-x}\left(\begin{array}{c}
c_{0}^{(n)^{\bullet}} \\
a_{0}^{(n)^{\bullet}}
\end{array}\right) k_{n}^{[-1]}\left(x k_{n} k_{p n}^{[-1]} a_{0}^{(n)},-c_{0}^{(n)}\right)\right)\left(\begin{array}{c}
p(z) \\
q(z)
\end{array}\right) h \\
= & \left(\begin{array}{c}
p(z) \\
a_{0}^{(n)^{\bullet}} k_{p n}^{[-1]} a_{0}^{(n)} p(z)+q(z)
\end{array}\right) h .
\end{aligned}
$$

By induction we obtain

$$
\left(\begin{array}{l}
p_{0}(z) \\
q_{0}(z)
\end{array}\right) h=\left(\sum_{l=0}^{n} a_{0}^{(l)} \underset{k_{p l}^{[-1]} a_{0}^{(l)} p(z)+q(z)}{p(z)}\right) h
$$

and, hence, (4.73) contradicts to (4.72).

Let, conversely, (4.72) be broken, and for any $z \in \mathbb{C}^{+}$there exists a vector $h \in \mathbb{C}^{m \times m}$ such that

$$
q(z) h=0 \quad \text { and } \quad a_{0}^{(l)} p(z) h=0 \quad(l=0, \ldots, n) .
$$

Therefore equalities (4.74) hold for $l=0, \ldots, n$ and after the inverse Schur process we obtain (4.75), which in view of (4.76) implies (4.73)

Now we can state the main result of this section.

Theorem 4.10. Let $A, C, K \geq 0$ and $K_{p} \geq 0$ be matrices defined by (2.9), (2.12), (2.19). Then the linear fractional transformation (9.16) with the resolvent matrix $\theta$ defined by (4.66) gives a parametrization of all the solutions to the problem(IS) when the parameter $\{p, q\}$ varies in $\overline{\mathbf{S}}_{m}^{0}$ and is of the form (4.67).

Corollary 4.11. Let $\operatorname{rank} A=m$. Then the problem $(I S)$ is solvable if and only if $K \geq 0$ and $K_{p} \geq 0$.

Proof. Since rank $A=m$, then $\overline{\mathbf{S}}_{m}^{0}=\overline{\mathbf{S}}_{m}$ and we use arguments from the proof of Corollary 4.8

The following example shows that conditions $K, K_{p} \geq 0$ alone do not ensure for the problem (IS) to be solvable.

Example 4.12. Let $s=2 ; m=3, n=0, x<0$ and let

$$
a_{0}=\left(\begin{array}{lll}
1 & 0 & 0 \\
1 & 1 & 0
\end{array}\right), \quad c_{0}=\left(\begin{array}{ccc}
-2 x & 2 x & 0 \\
0 & 0 & 1
\end{array}\right), \quad \gamma_{0}=\left(\begin{array}{ll}
1 & 0 \\
0 & 0
\end{array}\right) .
$$

Then, according to (2.12) and (2.13),

$$
K=\left(\begin{array}{ll}
1 & 0 \\
0 & 0
\end{array}\right) \geq 0 \quad \text { and } \quad K_{p}=\left(\begin{array}{cc}
-x & 0 \\
0 & 0
\end{array}\right) \geq 0
$$


and, therefore,

$$
P_{\text {Ker } K}=P_{\text {Ker } K,}=\left(\begin{array}{ll}
0 & 0 \\
0 & 1
\end{array}\right) \text {. }
$$

Let the problem (IS) with the interpolation data (4.77) has a solution. Then, in view of Theorem 4.10 , there exists a Stieltjes pair $\{p, q\}$ such that

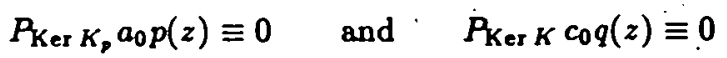

and

$$
\operatorname{det}\left(p(z)^{*} a_{0}^{*} a_{0} p(z)+q(z)^{*} q(z)\right) \not \neq 0 .
$$

Substituting (4.77), (4.79) into (4.80) we obtain $(1,1,0) p(z) \equiv 0$ and $(0,0,1) q(z) \equiv 0$. Applying Lemma 4.5 to the last identities we obtain that, up to equivalence,

$$
p(z)=\left(\begin{array}{cc}
\hat{p}(z) & 0 \\
0 & 1
\end{array}\right) \quad \text { and } \quad q(z)=\left(\begin{array}{cc}
\hat{q}(z) & 0 \\
0 & 0
\end{array}\right)
$$

Substituting (4.82) into (4.81) we obtain a contradiction. So, the problem (IS) has no solutions in spite of the non-negativity of $K, K_{p}$ which have been established in (4.78).

\section{References}

[1] Alpay, D., Ball, J., Gohberg, I. and L. Rodman: The two-sided residue interpolation in the Stieltjes class. Lin. Alg. Appl. (submitted).

[2] Alpay, D. and V. Bolotnikov: Two-sided Nevanlinna-Pick interpolation for a class of matriz-valued functions. Z. Anal. Anw. 12 (1993), 211 - 238.

[3] Alpay, D., Bruinsma, P., Dijksma, A. and H. de Snoo: Interpolation problems, extensions of symmetric operators and reproducing kernel spaces I. Oper. Theory: Adv. Appl. 50 (1991), 35 - 82.

[4] Alpay, D. and H. Dym: On applications of reproducing kernel spaces to the Schur algorithm and rational J-unitary factorization. Oper. Theory: Adv. Appl. 18 (1986), 89 - 159.

[5] Alpay, D. and H. Dym: On reproducing kernel spaces, the Schur algorithm and interpolation in a general class of domains. Oper. Theory: Adv. Appl. (to appear).

[6] Ball, J.: Interpolation problems of Pick-Nevanlinna and Leouner types for meromorphic matrix function. Int. Equ. Oper. Theory 6 (1983), $804-840$.

[7] Ball, J.: Nevanlinna-Pick interpolation: Generalizations and applications in: Surveys of some recent results in operator theory, volume 1 (J.B. Conway and B. B Morel Editor8), Pitman Research Notes in Mathematics: Vol. 171, (1989), 51 -94. Longman Scientfic and Technical Churchill Livingstone Inc. 1560 Broadway, New York.

[8] Ball, J., Gohberg, 1. and L. Rodman: Interpolation of Rational Matrix Functions (Opez. Theory: Adv. Appl.: Vol. 45). Basel et al: Birkhäuser Verlag 1990.

[9] Bolotnikov, V.: The two-sided Nevanlinna-Pick problem in the Stieltjes class. Oper. Theory: Adv. Appl. 62 (1993), 15 - 37.

[10] Bruinsma, P.: Degenerate interpolation problems for Nevanlinna pairs. Indag. Math. (to be published). 
[11] Dym, H.: J-contractive matrix functions, reproducing kernel Hilbert spaces and interpolation (Regional Conf. Series in Math.: Vol. 71). Providence (R. I.): Amer. Math. Soc. 1989.

[12] Dyukarev, Y. and V. Katsnelson: Multiplicative and additive classes of Stieltjes analytic matrix-valued functions and interpolation problems associated with them. Amer. Math. Soc. Transl. 131 (1986), $55 \cdot 70$.

[13] Efimov, A. and V. Potapov: J-expanding matrix functions and their role in the analytical theory of electrical circuits. Russian Math. Surveys 28 (1973), 69 - 140.

[14] Fedchina, I. : Tangential Nevalinna-Pick problem with multiple points. Doklady Acad. Nauk Arm. SSR 61 (1975), 214 - 218.

[15] Katsnelson, V.: Methods of J-theory in continuous interpolation problems of analysis. Private transl. by T.Ando, Sapporo, 1985.

[16] Kovalishina, I.: Analytic theory of a class of interpolation problems. Math. USSR Izv. 22 (1984), 419 - 463.

[17] Kovalishina, I. and V. Potapov: An indefinite metric in the Nevanlinna-Pick problem. Amer. Math. Soc. Transl. 138 (1988), $15-19$.

[18] Krein, M. and A. Nudelman: Markov Moment Problem and External Problems (Amer. Math. Soc. Transl. Math. Monographs: Vol. 50). Providence (R. I.): Amer. Math. Soc. 1977.

[19] Schur, I.: Über Protenzreihen, die im Innern des Einheitskreises beschränkt sind. J. Reine Ang. Math. 147 (1917), $205-232$.

Received 07.04.1993; in revised form 04.10.1993 